\title{
A retrospective evaluation of preoperative anemia in patients undergoing radical cystectomy for muscle-invasive urothelial urinary bladder cancer, with or without neoadjuvant chemotherapy
}

\author{
Gustaf Klinga and Amir Sherif
}

\begin{abstract}
Background and objective: Neoadjuvant chemotherapy (NAC) can be associated with anemia, which can lead to more perioperative blood transfusions (PBT). Usage of PBT is associated with worse oncological outcomes. We evaluated the prevalence of preoperative anemia (PA) and the effect on hemoglobin levels depending on surgery timing after NAC.

Methods: A retrospective single-center study with 240 consecutive patients undergoing radical cystectomy (RC) between 2001 and 2014 for muscle-invasive urothelial carcinoma (MIBC). Anemia was defined according to the WHO classification (male $\leq 130 \mathrm{~g} / \mathrm{L}$, female $\leq 120 \mathrm{~g} / \mathrm{L}$ ). Multivariable logistical regression was used to identify factors associated with PA and Pearson correlation for evaluating the change in hemoglobin levels depending on surgery timing.

Results: Overall, 128 (53.3\%) patients were anemic pre-RC and 87 (36.3\%) patients received NAC. In a multivariable analysis, age, receipt of NAC, female gender, and low BMI were independent predictors of PA. In patients receiving $N A C$, the time to surgery from the last NAC cycle was correlated with the change in hemoglobin levels between the initiation of NAC and surgery.
\end{abstract}

Conclusions: PA was common in patients undergoing RC for MIBC. Receipt of NAC was found to be a strong predictor of PA.

Clinical message: The emerging treatment of cisplatin based neoadjuvant chemotherapy for muscle-invasive bladder cancer, confers an increased risk for preoperative anemia. In the management of this malignancy, preoperative anemia renders further attention and focus.

Keywords: Urinary bladder neoplasms, Neoadjuvant therapy, Cisplatin, Anemia, Cystectomy

\section{Background}

Urothelial bladder cancer (UBC) is the fourth most common cancer in the Western World. The majority of patients diagnosed have non-invasive UBC, but around $25 \%$ are muscle-invasive (MIBC) at time of diagnosis (Burger et al. 2013). The recommended treatment

\footnotetext{
*Correspondence: amir.m.sherif@gmail.com; amir.sherif@urologi.umu.se Department of Surgical and Perioperative Sciences, Urology and Andrology, Umeå University, 90185 Umeå, Sweden
}

for patients with MIBC is radical cystectomy (RC) with lymph node dissection within 3 months to avoid progression and to decrease mortality. Addition of neoadjuvant chemotherapy (NAC) with cisplatin-based combinations is recommended for patients fit for chemotherapy (Witjes et al. 2015). This recommendation is based on the results of prospective randomized trials showing an absolute increase of survival with $5-8 \%, 5$ years median observation time (Sherif et al. 2004; Vale 2005). RC is 
considered major surgery and can be associated with significant blood loss and need for preoperative blood transfusions (PBT) (Shabsigh et al. 2009). The use of PBT have been shown to have a negative effect on prognosis in UBC (Abel et al. 2014; Linder et al. 2013; Gierth et al. 2014; Morgan et al. 2013). This detrimental effect has previously also been shown in other cancer types; gastric, colorectal and hepatocellular carcinoma (Ojima et al. 2009; Amato and Pescatori 2006; Wang et al. 2009). The mechanism of this unfavorable outcome is not fully understood. Negative immunomodulatory effects following transfusion have been suggested as one reason (Vamvakas and Blajchman 2007). Difficult surgical conditions in more advanced tumors or in patients with a history of abdominal surgery or radiation therapy of the pelvic organs, may also be a reason for increased use of PBT. Thus adding confounding factors to the long term results following PBT. Preoperative anemia (PA) has also been suggested to be an independent predictor for the outcome following RC. A recent study showed that PA is associated with worse oncological outcomes in patients undergoing RC (Gierth et al. 2015). In order to improve treatment in urothelial MIBC, it is valuable to have patients optimized prior to RC. The use of NAC has postulated side effects such as anemia and this, in combination with a risk of significant blood loss during surgery, can increase the risk for PBT. We sought to evaluate the prevalence of $\mathrm{PA}$, identify associated factors and evaluate the effect of NAC on hemoglobin $(\mathrm{Hb})$ levels in patients with urothelial MIBC undergoing RC.

\section{Methods}

In this retrospective single-center study we analyzed all RCs due to MIBC from 2001 to 2014. From the database we identified 349 consecutive patients, undergoing RC at a tertiary referral center (Norrland university hospital in Umeå, Sweden). We excluded 34 patients with nonurothelial cancer, 71 patients with non-invasive UBC and 4 patients who underwent salvage cystectomy. In summary, 240 consecutive patients constitute the study cohort. The study included 176 (73.3 \%) male and 64 (26.7\%) female patients and the median age was 69 years (IQR 62-75). Clinicopathological variables were gathered from patient journals regarding age, gender, BMI (Body Mass Index), ASA (American Society of Anesthesiologists) score, comorbidities (previous heart attack, hypertonia, atrial fibrillation, asthma/Chronic obstructive pulmonary disease (COPD), Diabetes Mellitus), hydronephrosis, estimated blood loss (EBL), receipt of PBT and number of units, receipt of NAC, clinical tumor stage (cT), Hb prior to first NAC-cycle, preoperative $\mathrm{Hb}$ and year of surgery.

Information about the change in $\mathrm{Hb}$ levels from the initiation of NAC until RC and also time to surgery from last NAC-cycle, was also evaluated. Hydronephrosis was defined as history of hydronephrosis prior to RC. PBT was defined as transfusion of allogenic red blood cells during the operation or in the postoperative hospitalization. The decision to administer blood transfusion was made by treating physicians. No standardized thresholds for transfusion were in place during the study period.

\section{Statistical analyses}

Patients were categorized based on their Hb-level into two groups: Normal Hb-level or anemic. Anemia was defined as $\mathrm{Hb} \leq 130 \mathrm{~g} / \mathrm{L}$ in male and $\mathrm{Hb} \leq 120 \mathrm{~g} / \mathrm{L}$ in female patients, according to the WHO classification (Blanc et al. 1968). Clinical and pathologic characteristics were compared between the groups using Mann-Whitney U test for continuous variables and Fisher's exact test for categorical variables. Spearman's rank correlation was used to check the development of EBL and usage of units of PBT over time. Univariable and multivariable logistic regression analysis was performed to identify factors associated with PA. In NAC-patients, Pearson correlation was used to analyze the relationship between the change in Hb-levels following NAC and time to RC. The reported $p$ values were two-sided and statistical significance was set at 0.05 . All statistical analyses were made using SPSS Statistics ${ }^{\circledR} 22$ (SPSS, IBM Corp, Armonk, NY, USA).

\section{Results}

Association of preoperative anemia with clinicopathologic variables

The median $\mathrm{Hb}$ was $125.5 \mathrm{~g} / \mathrm{L}$ (IQR 112.25-138.75). Overall, 128 (53.3\%) patients were anemic while 112 (46.7\%) patients were non-anemic. Anemic patients had an older age $(p=0.004)$, higher ASA-score $(p=0.024)$, a higher incidence of hydronephrosis $(p=0.003)$, received NAC $(p<0.001)$, lower EBL $(p=0.035)$, more PBT $(p=0.003)$, and higher cT $(p=0.016)$, compared to nonanemic patients. There was no significant difference in gender, BMI, history of heart attack, hypertonia, atrial fibrillation, asthma/COPD, Diabetes Mellitus or year of surgery (Table 1). Univariable analyses showed that age (Odds Ratio [OR] 1.046; $p=0.004$ ), hydronephrosis (OR 2.564; $p=0.003$ ), receipt of NAC (OR 6.744; $p<0.001$ ) and cT3 (OR 1.961; $p=0.041$ ) or cT4 stage (OR 4.814; $p=0.048)$, both compared to cT2 were associated with PA. ASA III-IV, female gender, history of heart attack, hypertonia, atrial fibrillation, Asthma/COPD, Diabetes Mellitus or BMI were not statistically significant. Multivariable analyses adjusted for the effects of age, gender, BMI, ASA, hydronephrosis, receipt of NAC and cT. Age (OR 1.071; $p=0.001$ ) and receipt of NAC (OR 9.668; $p<0.001)$ remained independent predictors of PA in the 
Table 1 Patient characteristics of the 240 patients with MIBC treated with radical cystectomy 2001-2014

\begin{tabular}{|c|c|c|c|c|}
\hline Parameter & All patients $(n=240)$ & Non-anemic $(n=112)$ & Anemic $(n=128)$ & $p$ value \\
\hline Age (years) & $69(62-75)$ & $68(61-73)$ & $71(65-76)$ & 0.004 \\
\hline Sex & & & & 0.145 \\
\hline Male & $176(73.3)$ & $77(68.8)$ & $99(77.3)$ & \\
\hline Female & $64(26.7)$ & $35(31.2)$ & $29(22.7)$ & \\
\hline BMI & $25.5(22.8-28.4)$ & $25.8(23.8-28.8)$ & $24.9(22.5-27.8)$ & 0.054 \\
\hline Missing data & 20 & 10 & 10 & \\
\hline ASA score & & & & 0.024 \\
\hline 1 & $28(11.8)$ & $19(17.3)$ & $9(7.1)$ & \\
\hline$\|$ & $120(50.6)$ & $57(51.8)$ & $63(49.6)$ & \\
\hline III & $86(36.3)$ & $32(29.1)$ & $54(42.5)$ & \\
\hline IV & $3(1.3)$ & $2(1.8)$ & $1(0.8)$ & \\
\hline Missing data & 3 & 2 & 1 & \\
\hline Prior heart attack & $33(13.8)$ & $15(13.4)$ & $18(14.1)$ & 1 \\
\hline Hypertonia & $104(43.3)$ & $51(45.5)$ & $53(41.4)$ & 0.602 \\
\hline Atrial fibrillation & $19(7.9)$ & $8(7.1)$ & $11(8.6)$ & 0.812 \\
\hline Asthma/COPD & $17(7.1)$ & $11(9.8)$ & $6(4.7)$ & 0.137 \\
\hline Diabetes mellitus & $29(12.1)$ & $13(11.6)$ & $16(12.5)$ & 0.845 \\
\hline Hydronephrosis & $63(26.3)$ & $19(17)$ & $44(34.4)$ & 0.003 \\
\hline Neoadjuvant chemotherapy & $87(36.3)$ & $17(15.2)$ & $70(54.7)$ & $<0.001$ \\
\hline $\mathrm{EBL}(\mathrm{mL})$ & $1000(700-1712.5)$ & $1200(725-2000)$ & $1000(650-1475)$ & 0.035 \\
\hline Missing data & 26 & 15 & 11 & \\
\hline Units of PBT & $4(2-6)$ & $2(1-6)$ & $4(2-6)$ & 0.003 \\
\hline cT & & & & 0.017 \\
\hline cT2 & $178(72.2)$ & $92(82.1)$ & $86(67.2)$ & \\
\hline cT3 & $51(21.3)$ & $18(16.1)$ & $33(25.8)$ & \\
\hline CT4 & $11(4.6)$ & $2(1.8)$ & $9(7.0)$ & \\
\hline Year of surgery & & & & 0.469 \\
\hline $2001-2002$ & $20(8.3)$ & $12(10.7)$ & $8(6.3)$ & \\
\hline $2003-2005$ & $43(17.9)$ & $21(18.8)$ & $22(17.2)$ & \\
\hline $2006-2008$ & $55(22.9)$ & $27(24.1)$ & $28(21.9)$ & \\
\hline 2009-2011 & $73(30.4)$ & $34(30.4)$ & $39(30.5)$ & \\
\hline 2012-2014 & 49 (20.4) & $18(16.1)$ & $31(24.2)$ & \\
\hline
\end{tabular}

Data are shown as median (IQR) or $n(\%)$

$B M I$ body mass index, $A S A$ American Society of Anesthesiologists, COPD chronic obstructive pulmonary disease, EBL estimated blood loss, $P B T$ perioperative blood transfusion, $C T$ clinical tumour stage

adjusted model, while hydronephrosis and cT did not. Female gender (OR 0.462; $p=0.046$ ) and BMI (OR 0.908; $p=0.022$ ) were found to be independent predictors in the adjusted model (Table 2). Further, there was no significant difference in PA comparing complete responders (pTONOM0) to non-responders or to progressing tumors $(p=0.778)$.

\section{Development of NAC, anemia, PBT and EBL over time}

The use of NAC increased during 2001-2014. The first patient receiving NAC was in 2003, with a steady increase during the years. In 2014, $81.3 \%$ of patients with MIBC received NAC at this center (Fig. 1a). There was no statistically significant increase in the prevalence of PA over time ( $p=0.067$; Fig. 1b). Overall, the median EBL was $1000 \mathrm{ml}$ (IQR 700-1712.5). There was no difference in EBL between patients receiving NAC compared to the non-NAC group $(p=0.369)$. The median EBL decreased from 2500 (IQR 2000-11,500) $\mathrm{ml}$ in 2001 to 875 (IQR 600-1050) $\mathrm{ml}$ in $2014(p=0.009)$. The median number of PBT was 4 units (IQR 2-6). No difference in the amount of PBT received was found between patients receiving NAC compared to the no-NAC group $(p=0.441)$. The median number of units transfused perioperatively declined from 7 units (IQR 2-11) in 2001 to 2.5 units (IQR $1-4)$ in 2014 ( $p=0.003$ ). Overall, 206 patients $(86.2 \%)$ received a PBT during hospitalization for RC. 
Table 2 Univariable and multivariable logistic regression analysis of risk factors associated with preoperative anemia

\begin{tabular}{|c|c|c|c|c|c|c|}
\hline \multirow[t]{2}{*}{ Variable } & \multicolumn{3}{|c|}{ Univariate } & \multicolumn{3}{|c|}{ Multivariate } \\
\hline & OR & $95 \% \mathrm{Cl}$ & $p$ value & OR & $95 \% \mathrm{Cl}$ & $p$ value \\
\hline Age & 1.046 & $1.015-1.078$ & 0.004 & 1.071 & $1.029-1.114$ & 0.001 \\
\hline \multicolumn{7}{|l|}{ Gender } \\
\hline Male & Referent & & & Referent & & \\
\hline Female & 0.644 & $0.363-1.146$ & 0.134 & 0.462 & $0.216-0.985$ & 0.046 \\
\hline BMI & 0.944 & $0.884-1.007$ & 0.081 & 0.908 & $0.837-0.986$ & 0.022 \\
\hline \multicolumn{7}{|l|}{ ASA score } \\
\hline I and || & Referent & & & Referent & & \\
\hline III and IV & 1.708 & $1.000-2.917$ & 0.050 & 1.761 & $0.886-3.500$ & 0.106 \\
\hline Hydronephrosis & 2.564 & $1.388-4.736$ & 0.003 & 1.761 & $0.829-3.778$ & 0.140 \\
\hline Reciept of NAC & 6.744 & $3.619-12.569$ & $<0.001$ & 9.668 & $4.456-20.480$ & $<0.001$ \\
\hline Prior heart attack & 1.058 & $0.506-2.213$ & 0.881 & & & \\
\hline Hypertonia & 0.845 & $0.507-1.410$ & 0.521 & & & \\
\hline Atrial fibrillation & 1.222 & $0.474-3.155$ & 0.678 & & & \\
\hline Asthma/COPD & 0.452 & $0.151-1.264$ & 0.130 & & & \\
\hline Diabetes mellitus & 1.088 & $0.499-2.374$ & 0.832 & & & \\
\hline \multicolumn{7}{|l|}{ сT } \\
\hline CT2 & Referent & & & Referent & & \\
\hline cT3 & 1.961 & $1.029-3.739$ & 0.041 & 1.781 & $0.767-4.135$ & 0.179 \\
\hline cT4 & 4.814 & $1.011-22.912$ & 0.048 & 2.878 & $0.506-16.378$ & 0.233 \\
\hline
\end{tabular}

OR odds ratio, $C I$ confidence interval, BMI body mass index, ASA American Society of Anesthesiologists, NAC neoadjuvant chemotherapy, COPD chronic obstructive pulmonary disease, $C T$ clinical tumor stage

\section{Effects of neoadjuvant chemotherapy on hemoglobin levels}

Table 3 shows the details of NAC treatment. In NACpatients $(n=87)$, the median time to surgery from the last cycle of NAC was 35 days (IQR 29-42). The median change in Hb-level was $-15 \mathrm{~g} / \mathrm{L}$ (IQR -26 to -3.5$)$. The change in Hb-levels can also be expressed in that the number of patients with anemia prior to NAC was 48 (55.2\%), compared to 70 (80.5\%) who had anemia prior to $\mathrm{RC}$ post-NAC. The change in $\mathrm{Hb}$ following NAC was correlated with time to $\mathrm{RC}$ (Pearson correlation coefficient: $0.221, p$ value: 0.042 , Fig. $2 \mathrm{a}$ ). There was an upward trend towards an increasing $\mathrm{Hb}$ after 7 weeks (Fig. 2b).

\section{Discussion}

The increased risk of adverse outcomes following PBT in conjunction with $\mathrm{RC}$ for $\mathrm{MIBC}$, needs to be addressed. Utilization of PBT affects the long term results, not only pertaining to overall and cancer-specific mortality but also to the long term risk of tumor recurrence (7). In general, PA increases the risk for usage of PBT, and we found it of importance to investigate effects of NAC on PA. This with background of optimizing this rapidly emerging treatment, which ultimately aims to improve both overall and cancer-specific mortality. We found that $53.3 \%$ of all the patients had PA. This is a higher prevalence than found in other studies, having reported rates ranging from 39.3 to 51.5 \% (Gierth et al. 2014, 2015; Moschini et al. 2015). However, two of the studies excluded patients receiving NAC and in the third study only $2.9 \%$ received NAC. We identified four independent predictors of PA: Age, gender, receipt of NAC and BMI. Receipt of NAC was the strongest predictor (OR 9.668). Anemia has previously been shown to be more common with increasing age (Gierth et al. 2015). A lower BMI could reflect the patient's state of health, which in turn can have an effect on Hb-levels. The fact that females had a lower risk of PA could partly be explained by a lower anemia threshold in the WHO-definition. Our current praxis is that $\mathrm{RC}$ is planned 4 weeks after final NAC-cycle. The planned time to $\mathrm{RC}$ in other centers in Sweden varies; some centers proceed after 6-8 weeks. The current EAUrecommendation regarding timing of $\mathrm{RC}$ is that the operation is not to be delayed more than 3 months. However, this recommendation is based on single-center studies on chemo naïve patients. A recent study explored the effect of delaying RC > 3 months on survival in a nationwide cohort (Bruins et al. 2016). The investigators also included NAC-patients, showing that the overall survival was similar between patients undergoing $\mathrm{RC}<3$ months compared to patients with $\mathrm{RC}>3$ months. This suggests that the 3-month recommendation may not be applicable 

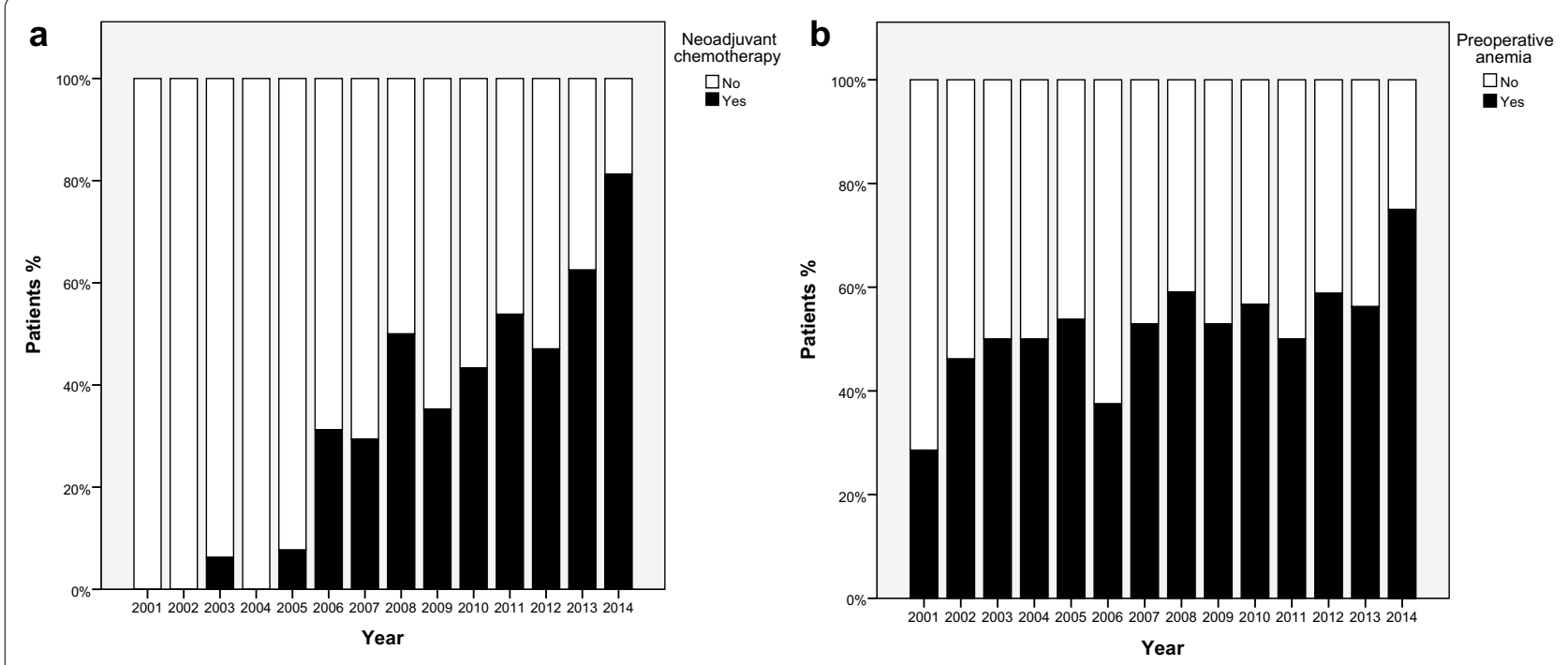

Fig. 1 a Use of neoadjuvant chemotherapy, 2001-2014. b Prevalence of preoperative anemia, 2001-2014. $p=0.067$

Table 3 Information about given NAC and hemoglobin values in the 87 patients who received NAC

\begin{tabular}{lc}
\hline Variable & Total $(\mathbf{n}=\mathbf{8 7})$ \\
\hline NAC treatment & \\
MVAC & $53(60.9)$ \\
HD-MVAC & $21(24.1)$ \\
Cisplatin-Gemzar & $6(6.9)$ \\
MVEC & $5(5.7)$ \\
Carboplatin-Gemzar & $1(1.1)$ \\
MVAC + Taxotere & $1(1.1)$ \\
Hemoglobin level (g/L) & \\
Prior to NAC & $127(117-127)$ \\
Prior to surgery & $115(107-123)$ \\
Anemic prior to NAC & $48(55.2)$ \\
Anemic prior to surgery & $70(80.5)$ \\
Number of days from last NAC to surgery & $35(29-42)$ \\
\hline
\end{tabular}

Data are shown as median (IQR) or $\mathrm{n}(\%)$

for NAC-patients. On the other hand, in NAC-nonresponders, an increased delay to $\mathrm{RC}$ might have a negative effect on survival. The problem with non-responders is currently being handled differently, depending on local traditions. Computerized tomography is commonly used in this center after the second NAC-cycle, for identifying non-responders and in case of non-response or progress, we proceed directly to RC avoiding NAC-cycle 3. Another suggested method is cystoscopy, with a recent study showing that cystoscopy-findings after NAC-cycle 2 are independent predictors of extravesical disease and pathologic downstaging (Mansour et al. 2015). In our study we have seen that utilization of NAC has increased in the last decade. Thus one can assume that with more patients receiving NAC, PA could become more prevalent. Another interesting approach would be to evaluate differences in PA, comparing complete responders to non-responders, thus postulating that micrometastic disease in the latter subgroup might be reflected in PA. Yet in this limited material there was no difference. Further we did not observe any differences in EBL or the amount of PBT received in the NAC group compared to the no-NAC-group. One reason might be that the NACpatients in our material were significantly younger than the chemo naive patients $(p=0.049)$. The amount of PBT decreased totally during the studied period. This is probably due to fewer surgeons performing $\mathrm{RC}$ following an ongoing national centralization of $\mathrm{RC}$, an increased awareness of both perioperative bleeding and EBL due to national registers (The Swedish bladder cancer register with increasing national coverage from the 2000s and the Swedish cystectomy register which started in 2011) and further the rapidly increasing use of ileal conduit $(86.5 \%$ for 2013 in Sweden) instead of other more time consuming urinary diversions.

Within the scope of prospective trials addressing these matters, consideration could be given to identify and treat anemia prior to $\mathrm{RC}$, specifically without allogenic blood transfusions. Another way to address the described problem could be in adjusting the time to surgery after $\mathrm{NAC}$, in order to let the Hb-level recover, and thus reduce the amount of patients with PA and the number of PBT required. In a few patients $(n=12)$ waiting for surgery more than 7 weeks after final NAC cycle, there 

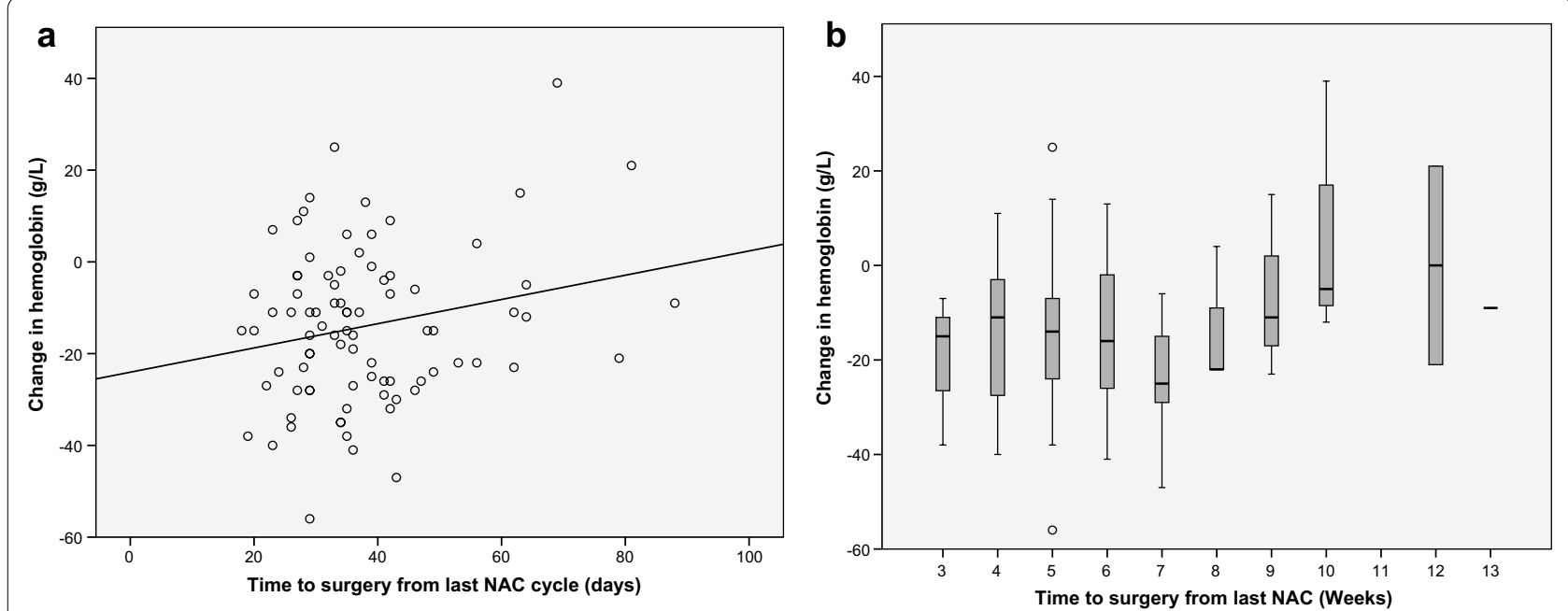

Fig. 2 a Correlation between time to surgery and change in hemoglobin following NAC. Pearson correlation coefficient: $0.221, p=0.042$. b Change in hemoglobin following NAC stratified over number of weeks to surgery

was a trend towards a smaller Hb-decrease post-NAC, compared with patients having a shorter interval to RC having a larger decrease. More data is needed to conclusively say if an increased time to surgery could affect the change in $\mathrm{Hb}$-levels. More studies are also required to assess if delayed surgery following NAC has an impact on survival. The limitations of this study include its retrospective design on a relatively small study population. No pathological review was made, and due to the long study period multiple surgeons were involved. Furthermore no data on GFR were available which could add additional information regarding selection of patients to NAC and the individual effect of NAC on renal function. More data from centers where the planned time to $\mathrm{RC}$ after NAC is longer could also add value to the analysis of the impact of delayed surgery on PA and the amount of PBT.

\section{Conclusions}

PA was common in patients undergoing RC for MIBC. Receipt of NAC was found to be a strong predictor of PA. This has not been described previously in any evaluations pertaining to NAC-treatment of urothelial MIBC. Strategies to identify and manage patients with PA should be developed to avoid PBT in order to optimize management and prognosis, yet both larger retrospective and also prospective evaluations relating to PA and NAC, need first to be undertaken.

\section{Authors' contributions}

GK: Data collection and management, Data analysis, Manuscript writing/ editing. AS: Protocol/project development, Data collection and management, Data analysis, Manuscript writing/editing. Both authors read and approved the final manuscript.

\section{Acknowledgements}

This work was supported by the Swedish Research Council funding for clinical research in medicine (ALF) in Västerbotten, VLL, Sweden. We also thank Johan Svensson from the Department of Statistics, Umeå University, Sweden, for his contribution to the statistical analysis.

\section{Competing interests}

The authors declare that they have no competing interests.

\section{Ethical standards}

The study was approved by the regional ethical committee (EPN Umeå, dnr: 2015/395-32).

Received: 20 May 2016 Accepted: 19 July 2016

Published online: 25 July 2016

\section{References}

Abel EJ, Linder BJ, Bauman TM, Bauer RM, Thompson RH, Thapa P et al (2014) Perioperative blood transfusion and radical cystectomy: does timing of transfusion affect bladder cancer mortality? Eur Urol 66(6):1139-1147 Amato A, Pescatori M (2006) Perioperative blood transfusions for the recurrence of colorectal cancer. Cochrane Database Syst Rev Jan(1):CD005033

Blanc B, Finch CAHL et al (1968) Nutritional anaemias. Report of a WHO scientific group. World Health Organ Tech Rep Ser 405:5-37

Bruins HM, Aben KKH, Arends TJ, van der Heijden AG, Witjes AJ (2016) The effect of the time interval between diagnosis of muscle-invasive bladder cancer and radical cystectomy on staging and survival: a Netherlands Cancer Registry analysis. Urol Oncol 34(4):166.e1-6

Burger M, Catto JWF, Dalbagni G, Grossman HB, Herr H, Karakiewicz P et al (2013) Epidemiology and risk factors of urothelial bladder cancer. Eur Urol 63(2):234-241

Gierth M, Aziz A, Fritsche HM, Burger M, Otto W, Zeman F et al (2014) The effect of intra- and postoperative allogenic blood transfusion on patients' survival undergoing radical cystectomy for urothelial carcinoma of the bladder. World J Urol 32(6):1447-1453

Gierth M, Mayr R, Aziz A, Krieger S, Wullich B, Pycha A et al (2015) Preoperative anemia is associated with adverse outcome in patients with urothelial carcinoma of the bladder following radical cystectomy. J Cancer Res Clin Oncol 141(10):1819-1826 
Linder BJ, Frank I, Cheville JC, Tollefson MK, Thompson RH, Tarrell RF et al (2013) The impact of perioperative blood transfusion on cancer recurrence and survival following radical cystectomy. Eur Urol 63(5):839-845

Mansour AM, Soloway MS, Eldefrawy A, Singal R, Joshi S, Manoharan M (2015) Prognostic significance of cystoscopy findings following neoadjuvant chemotherapy for muscle-invasive bladder cancer. Can J Urol 22(2):7690-7697

Morgan TM, Barocas DA, Chang SS, Phillips SE, Salem S, Clark PE et al (2013) The relationship between perioperative blood transfusion and overall mortality in patients undergoing radical cystectomy for bladder cancer Urol Oncol 31(6):871-877

Moschini M, Dell'Oglio P, Capogrosso P, Cucchiara V, Luzzago S, Gandaglia G et al (2015) Effect of allogeneic intraoperative blood transfusion on survival in patients treated with radical cystectomy for nonmetastatic bladder cancer: results from a single high-volume institution. Clin Genitourin Cancer. 13(6):562-567

Ojima T, Iwahashi M, Nakamori M, Nakamura M, Naka T, Katsuda M et al (2009) Association of allogeneic blood transfusions and long-term survival of patients with gastric cancer after curative gastrectomy. J Gastrointest Surg 13(10):1821-1830
Shabsigh A, Korets R, Vora KC, Brooks CM, Cronin AM, Savage C et al (2009) Defining early morbidity of radical cystectomy for patients with bladder cancer using a standardized reporting methodology. Eur Urol 55(1):164-174

Sherif A, Holmberg L, Rintala E, Mestad O, Nilsson J, Nilsson S et al (2004) Neoadjuvant cisplatinum based combination chemotherapy in patients with invasive bladder cancer: a combined analysis of two Nordic studies. Eur Urol 45(3):297-303

Vale CL (2005) Neoadjuvant chemotherapy in invasive bladder cancer: update of a systematic review and meta-analysis of individual patient data advanced bladder cancer (ABC) meta-analysis collaboration. Eur Urol 48(2):202-205; discussion 205-206

Vamvakas EC, Blajchman MA (2007) Transfusion-related immunomodulation (TRIM): an update. Blood Rev 21(6):327-348

Wang C-C, lyer SG, Low JK, Lin C-Y, Wang S-H, Lu S-N et al (2009) Perioperative factors affecting long-term outcomes of 473 consecutive patients undergoing hepatectomy for hepatocellular carcinoma. Ann Surg Oncol 16(7):1832-1842

Witjes JA, Compérat E, Cowan NC, De Santis M, Gakis G, James N et al (2015) Guidelines on muscle-invasive and metastatic bladder cancer. European Association of Urology

\section{Submit your manuscript to a SpringerOpen ${ }^{\circ}$ journal and benefit from:}

- Convenient online submission

- Rigorous peer review

- Immediate publication on acceptance

- Open access: articles freely available online

- High visibility within the field

- Retaining the copyright to your article 